ISSN 0103-5150

Fisioter. Mov., Curitiba, v. 24, n. 4, p. 647-654, out./dez. 2011

Licenciado sob uma Licença Creative Commons

\title{
Linfedema pós-câncer de mama: comparação de duas técnicas fisioterapêuticas - estudo piloto
}

\author{
Lymphedema after breast cancer: comparison between \\ two physical therapy techniques - a pilot study
}

\section{Nara Fernanda Braz da Silva Leal ${ }^{[a]}$, Letícia Alves Rios Dias ${ }^{[\mathrm{b}]}$, Hélio Humberto Angotti Carrara ${ }^{[\mathrm{c}]}$, Cristine Homsi Jorge Ferreira ${ }^{[\mathrm{d}]}$}

[a] Mestranda do Departamento de Ginecologia e Obstetrícia da Faculdade de Medicina de Ribeirão Preto da Universidade de São Paulo, Ribeirão Preto, SP - Brasil, e-mail: nara.leal@usp.br

[b] Mestre em Ciências da Reabilitação pela Faculdade de Medicina de Ribeirão Preto da Universidade de São Paulo, Ribeirão Preto, SP - Brasil, e-mail: leticia.alvesdias@yahoo.br

[c] Doutor e Professor do Departamento de Ginecologia e Obstetrícia da Faculdade de Medicina de Ribeirão Preto da Universidade de São Paulo, Ribeirão Preto, SP - Brasil, e-mail: hhacarra@fmrp.usp.br

[d] Doutora e Professora do Departamento de Biomecânica, Medicina e Reabilitação do Aparelho Locomotor da Faculdade de Medicina de Ribeirão Preto da Universidade de São Paulo, Ribeirão Preto, SP - Brasil, e-mail: cristine@fmrp.usp.br

\section{Resumo}

Introdução: 0 câncer de mama apresenta relevantes efeitos físicos e psicológicos. Entre as formas de tratamento, as cirurgias conservadoras, muitas vezes com esvaziamento linfático axilar e seguidas por radioterapia, têm papel preponderante. Uma importante complicação pós-tratamento é o surgimento do linfedema no membro superior homolateral. A principal terapêutica para o linfedema é a fisioterapia complexa descongestiva. A estimulação elétrica vem sendo utilizada na prática clínica para a redução do edema, sendo, por isso, uma nova alternativa para o tratamento do linfedema a ser pesquisada. Objetivo: Realizar um estudo piloto para comparar os efeitos da fisioterapia complexa descongestiva com um protocolo que inclui estimulação elétrica, exercícios terapêuticos e uso da braçadeira elástica na redução do linfedema secundário com o esvaziamento linfático axilar. Materiais e métodos: Doze voluntárias foram divididas aleatoriamente em dois grupos e submetidas a diferentes protocolos terapêuticos (FCD e EAV). A redução do linfedema foi avaliada por meio da perimetria e da volumetria de ambos os membros superiores. As intervenções foram realizadas duas vezes por semana por um período de sete semanas. Resultados: Não se observou diferença na redução do linfedema entre os protocolos utilizados. Conclusão: Com uma amostra pequena 
de pacientes, não se observaram diferenças entre as técnicas na redução do linfedema quando aplicadas na fase de manutenção. Fatores como tempo de instalação do linfedema, grau de esvaziamento axilar, radicalidade da cirurgia e aplicação de radioterapia na região afetada contribuem para o estágio do linfedema e sucesso de seu tratamento.

Palavras-chave: Câncer de mama. Linfedema. Estimulação elétrica. Fisioterapia.

\section{Abstract}

Introduction: Breast cancer is an illness with physical and psychological important effects. Its treatment forms include axillary dissection sugery. A considerable postoperative consequence is the upper limb lymphedema. The principal therapy of lymphedema is descongestive lymphatic therapy. Electrical stimulation has been applied clinically in reduction of edema. Due to this, it is a new alternative for lymphedema treatment to be studied. Objective: To compare the descongestive lymphatic therapy with a protocol that includes electrical stimulation, upper limb exercises and compression by bandages in the reduction of axillary dissection secondary lymphedema. Method: twelve volunteers were distributed randomly in the two groups and they were submitted to different treatments (FCD and EAV). The reduction of lymphedema was valued by perimetry and volumetry of upper limb. The interventions were realized twice a week during a period of seven weeks. Results: It did not reach statistic significance in reduction of edema. Conclusion: With a small sample of patients, there was no difference between the techniques in reducing lymphedema when applied to the maintenance phase. The time of installation of lymphedema, axillary dissection, sugery and radiotherapy contribute for degree of lymphedema and its treatment. The use of bandages compressive by the patients influences the success of therapy.

Keywords: Breast cancer. Lymphedema. Electrical stimulation. Physiotherapy.

\section{Introdução}

Segundo tipo mais frequente no mundo, o câncer de mama é o mais comum entre as mulheres, correspondendo a $22 \%$ dos casos novos a cada ano (1). Seu tratamento pode incluir radioterapia, quiomioterapia, hormonioterapia, cirurgia conservadora, mastectomias radical e radical modificada (2-5). Após a cirurgia e a excisão ou a radiação dos linfonodos axilares adjacentes, a paciente pode apresentar diversas complicações, entre as quais se destaca o linfedema $(3,6-8)$.

O linfedema é definido como acúmulo excessivo e persistente de fluido e proteínas extravasculares e extracelulares nos espaços teciduais por causa da ineficiência do sistema linfático $(3,9)$. Os fatores que podem influenciar no desenvolvimento do linfedema são: número de linfonodos removidos, radioterapia axilar, infecção na incisão cirúrgica, falta de mobilidade do membro superior e obesidade (10, 11). Os sinais e os sintomas associados ao linfedema são: aumento do diâmetro do membro, tensionamento da pele, rigidez, diminuição da amplitude de movimento (ADM) do membro e uso reduzido do membro em tarefas funcionais (3).

0 tratamento fisioterapêutico padrão para o linfedema é composto de duas fases: fase intensiva e fase de manutenção. A primeira fase é composta pela fisioterapia complexa descongestiva (FCD), técnica que combina drenagem linfática manual (DLM), enfaixamento compressivo funcional (ECF), exercícios terapêuticos, cuidados com a pele e precauções para a vida diária. Apresenta como objetivo a redução máxima do volume do membro com melhora estética e da funcionalidade. A duração da fase intensiva é determinada pela gravidade do caso, podendo variar de 3 semanas até meses, sendo concluída quando se atinge a redução máxima do volume do membro (parcial ou total). Já na fase de manutenção, que inicia com o fim da fase intensiva, visa a manter pelo máximo de tempo as reduções obtidas. Os recursos mais aplicados são: automassagem linfática, exercícios, uso da braçadeira e cuidados com a pele $(2,4,5,9)$.

A literatura demonstra que a FDC foi eficaz na redução do linfedema na fase intensiva. Essa redução manteve-se ao longo dos períodos estudados com a 
utilização de exercícios, automassagem, uso da braçadeira e cuidados com o braço (12). Um estudo prospectivo concluído com 62 mulheres obteve redução do volume e circunferência do membro acometido, além de melhora da qualidade de vida com a aplicação da FCD (13).

Como uma nova alternativa para o tratamento do linfedema, a estimulação elétrica tem sido proposta. Entre as formas de corrente, a estimulação de alta voltagem (EAV) apresenta indicação clínica para dores agudas e crônicas, aumento na velocidade da regeneração de tecidos, reeducação neuro-muscular, aumento do fluxo sanguíneo venoso e absorção de edema (14-16).

Uma pesquisa realizada com mulheres submetidas à mastectomia unilateral que apresentavam linfedema secundário no membro homolateral à cirurgia analisou o efeito da estimulação elétrica na redução desse edema. A avaliação do linfedema foi feita por meio da perimetria e da volumetria do braço e pelo cálculo da severidade relativa do linfedema. Concluiu que, após tratamento com EAV, houve diminuição da perimetria, da volumetria e da severidade do linfedema (17).

A fase intensiva do tratamento, que é administrada pelo profissional, apresenta redução importante do volume do membro acometido. Já a fase de manutenção, geralmente composta por técnicas de autoaplicação, traz menores reduções do volume do linfedema (18). Esses fatores, a escassez de estudos que investigaram opções para tratar o linfedema residual e os bons resultados recentemente descritos com o uso da corrente de alta voltagem justificam a relevância desta pesquisa. 0 objetivo deste trabalho foi comparar a efetividade de um protocolo tradicional de FCD com um protocolo que inclui a utilização da corrente de alta voltagem no tratamento do linfedema residual de membros superiores de mulheres submetidas ao esvaziamento linfático axilar e em fase de manutenção.

\section{Materiais e métodos}

\section{Pacientes}

Trata-se de um ensaio clínico piloto, realizado mediante aprovação do Comitê de Ética em Pesquisa da Escola de Enfermagem de Ribeirão Preto (EERP) (USP), de acordo com o protocolo número 0744/2006.

As voluntárias foram recrutadas e atendidas no Núcleo de Ensino, Pesquisa e Assistência na Reabi- litação de Mastectomizadas (REMA) da EERP-USP. Das 15 pacientes avaliadas inicialmente, 12 foram incluídas no estudo de acordo com os critérios de inclusão preestabelecidos. As voluntárias deveriam ter realizado esvaziamento linfático axilar unilateral, apresentar linfedema secundário ao tratamento no membro homolateral com diferença entre os braços encontrada na perimetria maior ou igual a $3 \mathrm{~cm}$ e aceitar participar do estudo mediante assinatura de um termo de consentimento livre e esclarecido. As voluntárias incluídas tinham sido submetidas ao tratamento do linfedema com FCD anteriormente e estavam na fase de manutenção.

Foram excluídas do estudo as pacientes com cirurgia bilateral, que apresentavam afecções da pele ou qualquer contraindicação ao uso de estimulação elétrica e DLM.

\section{Avaliação do linfedema}

A redução do linfedema foi analisada por meio de perimetria e volumetria. A perimetria seguiu a técnica com mensuração feita bilateralmente em seis pontos distintos: ponto $\mathrm{A}$ - na articulação metacarpofalangeana dos $2^{\circ}$, $3^{3}$, $4^{\circ}$ e e $5^{\circ}$ dedos; ponto $\mathrm{B}$ - uma linha imaginária passando na direção da articulação metacarpofalangeana do $1^{\mathrm{o}}$ dedo; ponto $\mathrm{C}-10 \mathrm{~cm}$ abaixo do olécrano; ponto $\mathrm{D}-6 \mathrm{~cm}$ abaixo do olécrano; ponto $\mathrm{E}-6 \mathrm{~cm}$ acima do olécrano; e ponto $\mathrm{F}-10$ cm acima do olecrano (19).

A volumetria foi realizada por um equipamento que consiste em um recipiente cilíndrico cheio de água, fechado em uma das extremidades e acoplado a um recipiente coletor graduado em mililitros. A paciente se posicionou sentada em um banco, com tornozelo em neutro e joelhos e quadris a $90^{\circ}$, e imergiu completamente um membro de cada vez até a altura do cavo axilar. 0 ângulo de inclinação do tronco foi registrado por um flexímetro. 0 volume de água extravasado foi registrado pelo recipiente coletor. As mensurações foram bilaterais e realizadas na $1^{\underline{a}} \mathrm{e}$ última sessões.

\section{Intervenção}

As pacientes foram divididas aleatoriamente em dois grupos de tratamento: grupo 1 - foi aplicada a FCD composta por cinesioterapia, drenagem linfática 
manual e uso de braçadeira; grupo 2 - foi aplicada a EAV associada à cinesioterapia e ao uso de braçadeira. Cada grupo foi composto por seis pacientes.

A estimulação elétrica apresentou os seguintes parâmetros: corrente pulsada de alta voltagem, monopolar (negativa), $50 \mathrm{~Hz}$, relação on/off de 3:9 segundos, rampa de subida/descida 2:1 segundos, modo de estimulação sincronizado e amplitude necessária para contração muscular e de acordo com a aceitação da voluntária (17). A duração da aplicação da corrente foi de 20 minutos utilizando o aparelho modelo Neurodyn High Volt ${ }^{\circledR}$ IBRAMED e os eletrodos foram posicionados dois a dois nas regiões anteriores do braço e do antebraço. A cinesioterapia para membro superior constou de exercícios ativos livres ou com uso de bastão, de punho, cotovelo e ombro, envolvendo flexão, extensão, adução, abdução e rotações interna e externa, realizados em grupo supervisionado por uma enfermeira e com duração de uma hora. A drenagem linfática manual teve duração de aproximadamente 50 minutos e a braçadeira foi de uso diário no membro afetado. Ambos os grupos receberam duas sessões por semana durante sete semanas. A aplicação da EAV e da DLM e as avaliações foram realizadas pela mesma fisioterapeuta.

\section{Análise estatística dos dados}

Para comparar a diferença nos valores da perimetria e da volumetria, tanto inter quanto intragrupo, foi utilizado o modelo de regressão linear com efeitos mistos (efeitos aleatórios e fixos). Os modelos lineares de efeitos mistos são utilizados na análise de dados em que as respostas estão agrupadas (medidas repetidas para um mesmo indivíduo) e a suposição de independência entre as observações num mesmo grupo não é adequada (20). Tal modelo tem como pressuposto que o resíduo obtido por meio da diferença entre os valores preditos pelo modelo e os valores observados tenha distribuição normal com média 0 e variância constante. 0 ajuste do modelo foi feito por meio do software SAS versão 9.0.

\section{Resultados}

Foram incluídas no presente estudo 12 voluntárias, entre as quais 9 concluíram todas as etapas do projeto, o tratamento e as duas avaliações. As carac- terísticas das voluntárias como idade, tempo de cirurgia e tratamento complementar à cirurgia estão apresentadas na Tabela 1. 0 tipo de cirurgia realizado pelas voluntárias está apresentado na Tabela 2 .

Os resultados referentes à perimetria foram obtidos pela média da diferença (membro acometido membro não acometido) ponto a ponto. Na análise intragrupo para o grupo 1 (FCD) os valores obtidos entre a $1^{\underline{a}}$ e $2^{\underline{a}}$ avaliação foram: ponto $A=1,25 \mathrm{~cm}$; ponto $\mathrm{B}=-0,13 \mathrm{~cm}$; ponto $\mathrm{C}=0,25 \mathrm{~cm}$; ponto $\mathrm{D}=$ $-0,13 \mathrm{~cm}$; ponto $\mathrm{E}=0,63 \mathrm{~cm}$ e ponto $\mathrm{F}=0 \mathrm{~cm}$. Já para o grupo 2 (EAV) foram: ponto $A=0,90 \mathrm{~cm}$; ponto $\mathrm{B}=$ $0,10 \mathrm{~cm}$; ponto $\mathrm{C}=-0,10 \mathrm{~cm}$; ponto $\mathrm{D}=0,20 \mathrm{~cm}$; ponto $\mathrm{E}=-0,20 \mathrm{~cm}$ e ponto $\mathrm{F}=0,70 \mathrm{~cm}$. Esses valores demonstraram que não houve diferença entre as avaliações 1 e 2 para ambos os grupos.

A análise intergrupo para a perimetria também foi realizada ponto a ponto considerando a diferença

Tabela $\mathbf{l}$ - Características das voluntárias de cada grupo

\begin{tabular}{lcc}
\hline Características & Grupo $\mathbf{1}(\mathbf{n}=6)$ & Grupo 2 $(\mathbf{n}=6)$ \\
\hline $\begin{array}{l}\text { Idade } \\
\text { (média) }\end{array}$ & 64 anos e 7 meses & 66 anos \\
$\begin{array}{l}\text { Tempo de cirurgia } \\
\text { (média) }\end{array}$ & 8 anos e 3 meses & 9 anos e 9 meses \\
$\begin{array}{l}\text { Quimioterapia } \\
\text { (n. de voluntárias) }\end{array}$ & 3 & 3 \\
$\begin{array}{l}\text { Radioterapia } \\
\text { (n. de voluntárias) }\end{array}$ & 6 & 5 \\
$\begin{array}{l}\text { Hormonioterapia } \\
\text { (n. de voluntárias) }\end{array}$ & 2 & 4 \\
\hline
\end{tabular}

Fonte: Dados da pesquisa.

Tabela 2 - Tipo de cirurgia realizado pelas voluntárias de cada grupo

\begin{tabular}{lcc}
\hline Voluntárias & Grupo $\mathbf{1}(\mathrm{n}=\mathbf{6})$ & Grupo 2 $(\mathrm{n}=\mathbf{6})$ \\
\hline Voluntária 1 & Quadrandectomia & Patey \\
Voluntária 2 & Halsted & Lumpectomia \\
Voluntária 3 & Quadrandectomia & Patey \\
Voluntária 4 & Patey & Quadrandectomia \\
Voluntária 5 & Halsted & Patey \\
Voluntária 6 & Patey & Patey \\
\hline
\end{tabular}

Fonte: Dados da pesquisa. 
(grupo1 - grupo 2). Os valores obtidos na avaliação 1 foram: ponto $\mathrm{A}=0,53 \mathrm{~cm}$; ponto $\mathrm{B}=0,35 \mathrm{~cm}$; ponto $\mathrm{C}=1,08 \mathrm{~cm}$; ponto $\mathrm{D}=0,43 \mathrm{~cm}$; ponto $\mathrm{E}=0,33 \mathrm{~cm}$ e ponto $\mathrm{F}=0,23 \mathrm{~cm}$. Na avaliação 2 , os valores encontrados foram: ponto $A=0,18 \mathrm{~cm}$; ponto $B=0,58$ $\mathrm{cm}$; ponto $\mathrm{C}=0,73 \mathrm{~cm}$; ponto $\mathrm{D}=0,75 \mathrm{~cm}$; ponto $\mathrm{E}=$ $-0,50 \mathrm{~cm}$ e ponto $\mathrm{F}=0,93 \mathrm{~cm}$. Esses resultados apontam que não houve diferença entre os tratamentos aplicados. A Tabela 3 apresenta as comparações em relação à perimetria.

Os resultados referentes à volumetria foram analisados por meio da média da diferença (membro acometido - membro não acometido). Os valores obti- dos para a análise intragrupo foram: grupo 1 (DLM) = $-42,75 \mathrm{ml}$ e grupo $2(\mathrm{EE})=-40,18 \mathrm{ml}$. A análise intergrupo, obtida pela diferença (grupo 1 - grupo 2), apresentou os valores: avaliação $1=115,77 \mathrm{ml}$ e avaliação $2=118,34 \mathrm{ml}$. Não houve diferença na volumetria entre ambos os grupos e ambas as avaliações. A Tabela 4 apresenta os valores em relação à volumetria.

\section{Discussão}

Os resultados do presente estudo demonstraram que não houve diferença entre as variáveis avaliadas

Tabela 3 - Comparações com relação à perimetria $(\mathrm{em} \mathrm{cm})$ - modelo de regressão linear com efeitos mistos

\begin{tabular}{|c|c|c|c|c|c|}
\hline \multirow[b]{2}{*}{ Ponto } & \multirow[b]{2}{*}{ Comparação } & \multirow{2}{*}{$\begin{array}{c}\text { Estimativa da } \\
\text { diferença }\end{array}$} & \multirow[b]{2}{*}{$\mathrm{p}$} & \multicolumn{2}{|c|}{ IC $(95 \%)$ para a diferença } \\
\hline & & & & LI & LS \\
\hline \multirow{4}{*}{ A } & Antes (FCD - EAV) & 0,53 & 0,70 & $-2,19$ & 3,24 \\
\hline & Depois (FCD - EAV) & 0,18 & 0,90 & $-2,54$ & 2,89 \\
\hline & FCD (Antes - Depois) & 1,25 & 0,15 & $-0,48$ & 2,98 \\
\hline & EAV (Antes - Depois) & 0,90 & 0,25 & $-0,65$ & 2,45 \\
\hline \multirow{4}{*}{ B } & Antes (FCD - EAV) & 0,35 & 0,80 & $-2,36$ & 3,06 \\
\hline & Depois (FCD - EAV) & 0,58 & 0,67 & $-2,14$ & 3,29 \\
\hline & FCD (Antes - Depois) & $-0,13$ & 0,89 & $-1,86$ & 1,61 \\
\hline & EAV (Antes - Depois) & 0,10 & 0,90 & $-1,45$ & 1,65 \\
\hline \multirow{4}{*}{ C } & Antes (FCD - EAV) & 1,08 & 0,43 & $-1,64$ & 3,79 \\
\hline & Depois (FCD - EAV) & 0,73 & 0,60 & $-1,99$ & 3,44 \\
\hline & FCD (Antes - Depois) & 0,25 & 0,77 & $-1,48$ & 1,98 \\
\hline & EAV (Antes - Depois) & $-0,10$ & 0,90 & $-1,65$ & 1,45 \\
\hline \multirow{4}{*}{ D } & Antes (FCD - EAV) & 0,43 & 0,76 & $-2,29$ & 3,14 \\
\hline & Depois (FCD - EAV) & 0,75 & 0,58 & $-1,96$ & 3,46 \\
\hline & FCD (Antes - Depois) & $-0,13$ & 0,89 & $-1,86$ & 1,61 \\
\hline & EAV (Antes - Depois) & 0,20 & 0,80 & $-1,35$ & 1,75 \\
\hline \multirow{4}{*}{$\mathrm{E}$} & Antes (FCD - EAV) & 0,33 & 0,81 & $-2,39$ & 3,04 \\
\hline & Depois (FCD - EAV) & $-0,50$ & 0,71 & $-3,21$ & 2,21 \\
\hline & FCD (Antes - Depois) & 0,63 & 0,47 & $-1,11$ & 2,36 \\
\hline & EAV (Antes - Depois) & $-0,20$ & 0,80 & $-1,75$ & 1,35 \\
\hline \multirow{4}{*}{$\mathrm{F}$} & Antes (FCD - EAV) & 0,23 & 0,87 & $-2,49$ & 2,94 \\
\hline & Depois (FCD - EAV) & 0,93 & 0,50 & $-1,79$ & 3,64 \\
\hline & FCD (Antes - Depois) & 0,00 & 0,99 & $-1,73$ & 1,73 \\
\hline & EAV (Antes - Depois) & 0,70 & 0,37 & $-0,85$ & 2,25 \\
\hline
\end{tabular}

Fonte: Dados da pesquisa. 
Tabela 4 - Comparações com relação à volumetria (em ml) - modelo de regressão linear com efeitos mistos

\begin{tabular}{lcccc}
\hline & \multirow{2}{*}{$\begin{array}{c}\text { Estimativa da } \\
\text { diferença }\end{array}$} & p & \multicolumn{2}{c}{ IC (95\%) para a diferença } \\
\cline { 4 - 5 } Comparação & 115,77 & 0,66 & LI & LS \\
\hline Antes (FCD - EAV) & 118,34 & 0,66 & $-487,36$ & 718,9 \\
Depois (FCD - EAV) & $-42,75$ & 0,60 & $-227,28$ & 721,47 \\
\hline FCD (Antes - Depois) & $-40,18$ & 0,58 & $-205,23$ & 141,78 \\
EAV (Antes - Depois) & & &
\end{tabular}

Fonte: Dados da pesquisa.

ao se comparar duas formas distintas de tratamento para o linfedema residual.

O linfedema do membro superior é uma complicação importante do tratamento para o câncer de mama, e com o aumento da sobrevida das mulheres com esse tipo de câncer, torna-se um problema crônico (21). 0 resultado da terapia para redução do linfedema depende de vários fatores. Uma vez instalado, o linfedema pode ser controlado, porém não curado (22-24). 0 linfedema pode ser reduzido significativamente na primeira semana de tratamento, sendo que, após a terceira semana, a redução pode ocorrer de maneira pouco significativa $(12,25)$. A partir desse momento, o tratamento deve ser continuado para uma fase de manutenção da redução já conseguida anteriormente $(23,26)$. Acredita-se que os resultados mais satisfatórios são obtidos quando o tratamento é iniciado assim que os primeiros sinais de linfedema aparecem $(22,27)$. Mesmo assim, ainda é sugerido que a repetição periódica da fase intensiva de tratamento possa melhorar os resultados obtidos nessa fase (12).

Com uma amostra final de nove voluntárias, os resultados encontrados neste estudo piloto não foram significativos para a redução do linfedema. Apesar de não haver redução, a estabilização do linfedema também é importante, pois com o não aumento das medidas, pode-se manter a função do membro e reduzir a incidência de infecção recorrente (26).

A literatura apresenta estudos utilizando todos os componentes da FDC, de parte deles ou ainda a substituição de algum deles por outra técnica. Um recente estudo, que aplicou FDC na fase intensiva, apresentou como objetivo avaliar o volume do linfedema durante a fase de manutenção e determinar os fatores que o influenciam. Foi concluído que o uso da braçadeira e o enfaixamento estão associados a melhores resultados e o insucesso da fase de manutenção está relacionado com a menor idade e altos índices de massa corpórea (28). Outro estudo comparou FCD + compressão pneumática (CP) com FCD modificada (substituição da bandagem compressiva pela Kinesio tape) + CP e obteve resultados satisfatórios com a substituição da FDC tradicional pela modificada (29).

0 acréscimo da DLM a uma terapia (exercícios, cuidados com a pele e uso da braçadeira) não trouxe evidência de melhores efeitos sobre o linfedema (30), e quando diferentes técnicas foram comparadas (FDC, DLM e automassagem + exercícios), não houve diferença entre elas em relação à redução do linfedema (21). Em outra pesquisa, houve aumento do volume do linfedema durante a fase de manutenção após redução com FCD na fase intensiva. Os autores atribuem esse aumento à não adesão ao uso da compressão elástica pelas pacientes (26).

Os estudos realizados somente com EAV para o tratamento do linfedema mostram redução satisfatória da perimetria e da volumetria do membro acometido $(17,22)$. A comparação da EAV com DLM + braçadeira apresentou redução maior da volumetria com a EAV (31). A aplicação da EAV + exercícios + automassagem + orientações de cuidados com o membro foi eficaz na redução do linfedema (32).

0 presente estudo apresenta diferenças em relação aos anteriormente citados: maior tempo decorrente desde a cirurgia e de instalação do linfedema e o fato de as voluntárias estarem na fase de manutenção do tratamento. Esses fatores podem justificar a manutenção das medidas do membro acometido encontrada neste estudo, talvez sendo o mais relevante por as pacientes já terem realizado tratamento prévio com fisioterapia complexa descongestiva (fase intensiva). Este parece ser o primeiro estudo que investiga 
a EAV como opção terapêutica para o linfedema residual, isto é, na fase de manutenção. Apesar de a nossa pesquisa ter sido controlada e ter utilizado métodos de avaliação confiáveis, há necessidade de uma ampliação dessa amostra, de modo a consubstanciar esses achados.

\section{Conclusão}

Esta pesquisa traz como conclusão que tanto a fisioterapia complexa descongestiva quanto o protocolo com estimulação elétrica não foram efetivos na redução do linfedema residual secundário ao esvaziamento linfático axilar. Entretanto, proporcionaram a manutenção das medidas avaliadas.

\section{Agradecimentos}

Agradecemos ao apoio financeiro recebido pela Fundação de Amparo à Pesquisa do Estado de São Paulo (Fapesp) processo n. 07/50115-8, à EERPUSP e ao REMA pela autorização e colaboração para realizar essa pesquisa.

\section{Referências}

1. Instituto Nacional do Câncer (INCa/MS). [acesso 21 set. 2010]. Disponível em: htpp://www.inca.gov.br.

2. Camargo MC, Marx AG. Reabilitação física no câncer de mama. São Paulo: Roca; 2000.

3. Kisner C, Colby LA. Tratamento de distúrbios vasculares dos membros. In: Kisner C, Colby LA. Exercícios terapêuticos fundamentos e técnicas. São Paulo: Manole; 2005. p.708-30.

4. Guirro E, Guirro R. Mastectomia. In: Guirro E, Guirro R. Fisioterapia dermato-funcional: fundamentos, recursos, patologias. São Paulo: Manole; 2002.

5. Silva ICA, Moraes V. Terapêutica em mastectomia. In: Borges FS. Dermato-funcional: modalidades terapêuticas nas disfunções estéticas. São Paulo: Phorte; 2006.

6. Panobianco MS, Mamede MV. Complicações e intercorrências associadas ao edema de braço nos três primeiros meses pós mastectomia. Rev Latino-Am Enfermagem. 2002;10(4):544-51.
7. Silva MPP, Derchain SFM, Rezende L, Cabello C, Martinez EZ. Movimento do ombro após cirurgia por carcinoma invasor da mama: estudo randomizado prospectivo controlado de exercícios livre versus limitados a $90^{\circ}$ no pós-operatório. RBGO. 2004;26(2):125-30.

8. Rezende LF, Beletti PO, Franco RL, Moraes SS, Gurge MSC. Exercícios livres versus direcionados nas complicações pós-operatórias de câncer de mama. Rev Assoc Med Bras. 2006;52(5):337-41.

9. Andrade MFC. Linfedema. In: Pitta GBB, Castro AA, Burihan E. Angiologia e cirurgia vascular: guia ilustrado. Maceió: UNICISAL/E CM AL \& LAVA; 2003.

10. Shaw C, Mortimer P, Judd PA. Randomized controlled trial comparing a low-fat diet with a weight-reduction diet in breast câncer-related lymphedema. Cancer. 2007;109(10):1949-56.

11. Harris SR, Hugi MR, Olivotto IA, Levine M. Clinical practice guidelines for the care and treatment of breast câncer: 11. CM AJ. 2001;164(2):191-9.

12. Meirelles MCCC, Mamede MV, Souza L, Panobianco MS. Avaliação de técnicas fisioterapêuticas no tratamento do linfedema pós-cirurgia de mama em mulheres. Rev Bras Fisioter. 2006;10(4):393-9.

13. Karadibak D, Yavuzsen T, Saydam S. Prospective trial of intensive decongestive physiotherapy for upper extremity lymphedema. J Surg Oncol. 2008;97(7):572-7.

14. Along G. High voltage stimulation: a monograph. Chattanooga, TN: Chattanooga Corporation; 1984.

15. Davini R, Nune CV, Guirro ECO, Guirro RRJ. Estimulação elétrica de alta voltagem: uma opção de tratamento. Rev Bras Fisioter. 2005;9(3):249-56.

16. Alon G. Os princípios da estimulação elétrica. In: Nelson RM, Hayes KW, Currier DP. Eletroterapia clínica. Barueri: Manole; 2003. p. 55-139.

17. Garcia LB, Guirro ECO. Efeitos da estimulação de alta voltagem no linfedema pós-mastectomia. Rev Bras Fisioter. 2005;9(2):243-8.

18. Mosely AL, Carati CJ, Piller NB. A systematic review of common conservative therapies for arm lymphoedema secondary to breast cancer treatment. Ann Oncol. 2007;18(4):639-46.

19. Mamede MV. Reabilitação de mastectomizadas: um novo enfoque assistencial [tese]. São Paulo: Universidade de São Paulo; 1991. 
20. Schall R. Estimation in generalized linear models with random effects. Biometrika. 1991;78(4):719-27.

21. Koul R, Dufan T, Russell C, Guenther W, Nugent Z, Sun X, et al. Efficacy of complete decongestive therapy and manual lymphatic drainage on treatmentrelated lymphedema im breast cancer. Int J Radiat Oncol Biol Phys. 2007;67(3):841-6.

22. Garcia LB, Guirro ECO, Montebello MIL. Efeitos da estimulação elétrica de alta voltagem no linfedema pós-mastectomia bilateral: estudo de caso. Fisioter Pesqui. 2007;14(1):67-71.

23. Mondry TE, Johnstone PAS. Manual lymphatic drainage for lymphedema limited to the breast. J Surg Oncol. 2002;81(2):101-4.

24. Brennan MJ, Miller LT. Overview of treatment options and review of the current role and use of compression garments, intermittent pumps, and exercise in the management of lymphedema. Cancer. 1998;83(12 Suppl):2821-7.

25. Leduc O, Leduc A, Bourgeois P, Belgrado JP. The physical treatment of upper limb edema. Cancer. 1998;83 (12 Suppl):2835-9.

26. Vignes S, Porcher R, Arraul M, Dupuy A. Long-term management of breast cancer-related lymphedema after intensive decongestive physiotherapy. Breast Cancer Res Treat. 2007;101(3):285-90.

27. Hammer JB, Fleming MD. Lymphedema therapy reduces the volume of edema and pain in patients with breast cancer. Ann Surg Oncol. 2007;14(6):1904-8.
28. Vignes S, Porcher R, Arraul M, Dupuy A. Factors influencing breast cancer-related lymphedema volume after intensive decongestive physiotherapy. Support Care Cancer. 2011;19(7):935-40.

29. Tsai H, Hung H, Yang J, Huang C, Tsauo J. Could Kinesio tape replace the bandage in decongestive lymphatic therapy for breast-cancer-related lymphedema? A pilot study. Support Care Cancer. 2009;17(11):1353-60.

30. Andersen L, Hojris I, Erlandsen M, Andersen J. Treatment of breast-cancer-related- lymphedema with or without manual lymphatic drainage. A randomized study. Acta Oncol. 2000;39(3):399-405.

31. Garcia LB, Guirro ECO, Montebello MIL. Avaliação de diferentes recursos fisioterapêuticos no controle do linfedema pós-mastectomia. Rev Bras Mastol. 2005; 15(2):64-70.

32. Barros VM, Panobianco MS. Linfedema pós-câncer de mama: protocolo de tratamento com estimulação elétrica de alta voltagem. [dissertação]. São Paulo: Universidade de São Paulo; 2009.

Recebido: $31 / 10 / 2010$ Received: $10 / 31 / 2010$

Aprovado: 18/05/2011 Approved: 05/18/2011 